\title{
Determining the 3D Atomic Coordinates and Crystal Defects in 2D Materials with Picometer Precision
}

Xuezeng Tian ${ }^{1}$, Dennis S. Kim ${ }^{1}$, Shize Yang ${ }^{2,3}$, Christopher J. Ciccarino ${ }^{4,5}$, Yongji Gong ${ }^{6}$, Yongsoo Yang ${ }^{1,7}$, Yao Yang ${ }^{1}$, Blake Duschatko ${ }^{4}$, Yakun Yuan ${ }^{1}$, Pulickel M. Ajayan ${ }^{6}$, Juan-Carlos Idrobo ${ }^{2}$, Prineha Narang ${ }^{4}$ and Jianwei Miao ${ }^{1 *}$

1. Department of Physics \& Astronomy and California NanoSystems Institute, University of California, Los Angeles, CA.

2. Center for Nanophase Materials Sciences, Oak Ridge National Laboratory, Oak Ridge, TN.

3. Center for Functional Nanomaterials, Brookhaven National Laboratory, Upton, NY.

4. John A. Paulson School of Engineering and Applied Sciences, Harvard University, Cambridge, MA.

5. Department of Chemistry and Chemical Biology, Harvard University, Cambridge, MA.

6. Department of Mechanical Engineering \& Materials Science, Rice University, Houston, Texas.

7. Department of Physics, Korea Advanced Institute of Science and Technology, Daejeon 34141, Korea.

* Corresponding author: miao@physics.ucla.edu

The field of research in two-dimensional (2D) materials, which possess valuable properties for many applications, has experienced extraordinary growth during the past decade $[1,2]$. To understand the structure-property relationship of 2D materials and heterostructures at the fundamental level, we must know their 3D atomic structure and crystal defects with high precision. Here, we developed scanning atomic electron tomography (sAET) to localize the 3D atomic coordinates in $2 \mathrm{D}$ materials and heterostructures with picometer precision. AET allows the determination of 3D atomic structure of crystal defects and disorder systems [4-9], and has recently been advanced to capture nucleation at 4D atomic resolution [10]. However, AET has thus far been limited to metallic nanoparticles and needleshaped samples. Application of AET to 2D materials and heterostructures would open a new frontier in 3D atomic structure characterization, but requires overcoming three obstacles. First, these materials are electron beam sensitive and the experiments must be performed with low electron doses. Second, the 3D precision of experimental atomic coordinates must be on the picometer scale so that they can be used as direct input to quantum mechanical calculations to determine physical, material and electronic properties. Third, due to the geometric constraint of 2D materials and heterostructures, the tilt range of data acquisition is limited by the reduced depth of focus of aberration-corrected electron microscopy.

Using a Re-doped $\mathrm{MoS}_{2}$ monolayer, we demonstrated a general sAET method to overcome these limitations and determined the 3D coordinates of individual atoms with high precision. We identified 3D crystal defects such as dopants, vacancies and atomic-scale ripples and measured the 3D atomic displacement and the full strain tensor of the 2D material. Furthermore, the experimental 3D atomic coordinates were used as direct input to DFT to correlate crystal defects with the electronic band structure at the single-atom level. We observed stark differences between the band structures obtained from the experimental and relaxed atomic models. We anticipate that sAET is not only generally applicable to the determination of the $3 \mathrm{D}$ atomic coordinates of $2 \mathrm{D}$ materials, heterostructures and thin films, but also could transform ab initio calculations by using experimental atomic coordinates as direct input to reveal more realistic physical, material, chemical and electronic properties [12]. 
References:

[1] A Geim, I Grigorieva, Nature 499 (2013), p. 419.

[2] K Novoselov et al., Science, 353 (2016), p. 6298.

[3] J Miao et al., Science 353 (2016), p. 6306.

[4] M C Scott et al., Nature 483 (2012), p. 444.

[5] B Goris et al., Nano Lett. 13 (2013), p. 4236.

[6] C Chen et al., Nature 496 (2013), p. 74.

[7] G Haberfehlner et al., Nat. Commun. 6 (2015), p. 8779.

[8] R Xu et al., Nat. Mater. 14 (2015), p. 1099.

[9] Y Yang et al., Nature 542 (2017), p. 75.

[10] J Zhou et al., arXiv:1807.10709 (2018).

[11] Y Yang et al., Nature 542 (2017), p. 75.

[12] This work was supported by the Office of Basic Energy Sciences of the US DOE (DE-SC0010378), STROBE: A National Science Foundation Science \& Technology Center (DMR-1548924), the Division of Materials Research of the US NSF (DMR-1437263), by an Army Research Office MURI grant on Ab-Initio Solid-State Quantum Materials: Design, Production, and Characterization at the Atomic Scale (18057522), and by the Center for Nanophase Materials Sciences, which is a Department of Energy Office of Science User Facility (JCI).

A

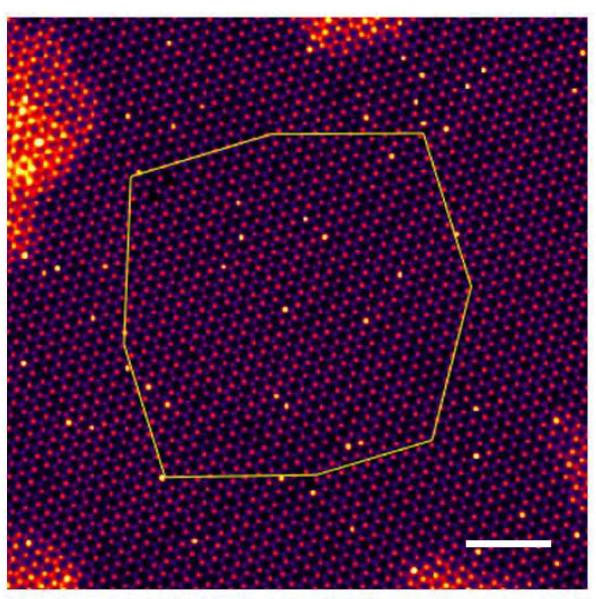

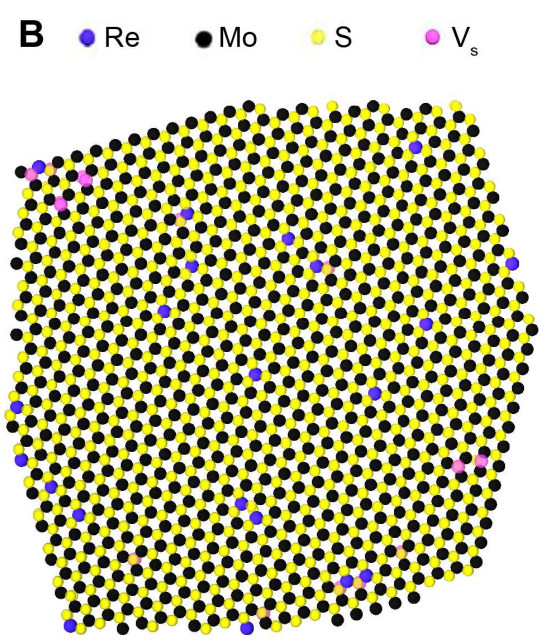

Figure 1. Example of reconstruction of 2D materials. (A) STEM image single layer Re-doped $\mathrm{MoS}_{2}$. Polygon shows the region of interest. Scale bar: $2 \mathrm{~nm}$. (B) 3D atomic structure of the region in (A). (C) Displacement map of the Mo-Re layer showing the non-flatness of the sample with picometre precision. 